\title{
Global Exponential Stability of a Unique Almost Periodic Solution for Neutral-Type Cellular Neural Networks with Distributed Delays
}

\author{
Wenquan Wu \\ Department of Mathematics, Aba Teachers College, Wenchuan, Sichuan 623002, China \\ Correspondence should be addressed to Wenquan Wu; mathwqwu@126.com
}

Received 4 January 2014; Revised 15 April 2014; Accepted 15 April 2014; Published 13 May 2014

Academic Editor: Naseer Shahzad

Copyright (C) 2014 Wenquan Wu. This is an open access article distributed under the Creative Commons Attribution License, which permits unrestricted use, distribution, and reproduction in any medium, provided the original work is properly cited.

This paper is concerned with the problem of the existence, uniqueness, and global exponential stability for neutral-type cellular neural networks with distributed delays. Based on fixed point theory and Lyapunov functional, several sufficient conditions are established for the existence, uniqueness, and global exponential stability of almost periodic solution for the above system. Finally, a simple example is given to illustrate the feasibility and effectiveness of our main results.

\section{Introduction}

It is well known that the delayed cellular neural networks (DCNNs) have been successfully applied to many practical problems, such as signal and image processing, pattern recognition, and optimization. Hence, they have been investigated extensively by many researchers in recent years. In particular, the stability and the existence of almost periodic solutions are two important properties, which have close relation to the applications of neural networks, so they have been widely studied. Therefore, there have been extensive results on the existence and stability of periodic solutions and almost periodic solutions of delayed cellular neural networks in the literature. We refer the reader to [1-12] and the references cited therein for more details.

As pointed out in [13], in the biochemistry experiments, neural information may transfer across chemical reactivity, which results in a neutral-type process. Moreover, in many cases the existing neural network models cannot characterize the properties of a neural reaction process precisely. To solve this problem, it is naturally introducing the neutral networks. To the best of our knowledge, however, the problem of global exponential stability of almost periodic solution for neutraltype cellular neural networks has not been fully investigated in the literature.
In this paper, we consider the following neutral-type cellular neural networks with distributed delays:

$$
\begin{aligned}
\dot{x}_{i}(t)= & -a_{i}(t) x_{i}(t)+\sum_{j=1}^{n} b_{i j}(t) f_{j}\left(x_{j}(t)\right) \\
& +\sum_{j=1}^{n} c_{i j}(t) g_{j}\left(\int_{0}^{\infty} w_{j}(s) x_{j}(t-s) \mathrm{d} s\right) \\
& +\sum_{j=1}^{n} d_{i j}(t) h_{j}\left(\int_{0}^{\infty} v_{j}(s) \dot{x}_{j}(t-s) \mathrm{d} s\right)+I_{i}(t),
\end{aligned}
$$

where $n$ denotes the number of units in a neural network, $x_{i}(t)$ corresponds to the state of the $i$ th unit at time $t, a_{i}>0$ represents the passive decay rates at time $t, f_{j}, g_{j}$, and $h_{j}$ are the neuronal output signal functions, $a_{i j}(t), b_{i j}(t), c_{i j}(t)$, and $d_{i j}(t)$ denote the strength of the $j$ th unit on the $i$ th unit at time $t, I_{i}(t)$ denotes the external inputs at time $t$, and $a_{i}$, $a_{i j}, b_{i j}, c_{i j}, d_{i j}$, and $I_{i}$ are all almost periodic functions, $i, j=$ $1,2, \ldots, n$. The kernel functions $w_{j}, v_{j}:[0,+\infty) \rightarrow[0,+\infty)$ are continuous with $\int_{0}^{\infty} w_{j}(s) \mathrm{d} s=\int_{0}^{\infty} v_{j}(s) \mathrm{d} s=1$ and satisfy

$$
\int_{0}^{\infty} e^{\xi s} w_{j}(s) \mathrm{d} s=p_{j}(\xi), \quad \int_{0}^{\infty} e^{\xi s} v_{j}(s) \mathrm{d} s=q_{j}(\xi),
$$


where $p_{j}$ and $q_{j}$ are continuous functions in $[0, \delta](\delta>0)$ and $p_{j}(0)=q_{j}(0)=1, j=1,2, \ldots, n$.

Let $C(\mathbb{X}, \mathbb{Y})$ and $C^{1}(\mathbb{X}, \mathbb{Y})$ be the space of continuous functions and continuously differential functions which map $\mathbb{X}$ into $\mathbb{Y}$, respectively. Especially, $C(\mathbb{X}):=C(\mathbb{X}, \mathbb{X}), C^{1}(\mathbb{X}):=$ $C^{1}(\mathbb{X}, \mathbb{X})$. For any bounded function $f \in C(\mathbb{R}), f^{+}=$ $\sup _{s \in \mathbb{R}}|f(s)|, f^{-}=\inf _{s \in \mathbb{R}}|f(s)|$. form

The initial conditions associated with system (1) are of the

$$
\begin{gathered}
x_{i}(s)=x_{i}^{*}(s), \quad \forall s \in(-\infty, 0], \\
x_{i}^{*} \in C^{1}((-\infty, 0], \mathbb{R}), \quad i=1,2, \ldots, n .
\end{gathered}
$$

We list some assumptions which will be used in this paper.

$\left(H_{1}\right) \min _{1 \leq i \leq n}\left\{a_{i}^{-}\right\}>0$.

$\left(H_{2}\right)$ There exist some positive constants $L_{j}^{f}, L_{j}^{g}$, and $L_{j}^{h}$ such that

$$
\begin{aligned}
& \left|f_{j}(u)-f_{j}(v)\right| \leq L_{j}^{f}|u-v|, \\
& \left|g_{j}(u)-g_{j}(v)\right| \leq L_{j}^{g}|u-v|, \\
& \left|h_{j}(u)-h_{j}(v)\right| \leq L_{j}^{h}|u-v|,
\end{aligned}
$$

for all $u, v \in \mathbb{R}, j=1, \ldots, n$.

$\left(H_{3}\right) \theta=\max \left\{\theta_{1}, \theta_{2}\right\}<1$, where

$$
\begin{gathered}
\theta_{1}=\max _{1 \leq i \leq n}\left\{\left(a_{i}^{-}\right)^{-1} \sum_{j=1}^{n}\left(b_{i j}^{+} L_{j}^{f}+c_{i j}^{+} L_{j}^{g}+d_{i j}^{+} L_{j}^{h}\right)\right\}, \\
\theta_{2}=\max _{1 \leq i \leq n}\left\{\left[1+\frac{a_{i}^{u}}{a_{i}^{l}}\right] \sum_{j=1}^{n}\left(b_{i j}^{+} L_{j}^{f}+c_{i j}^{+} L_{j}^{g}+d_{i j}^{+} L_{j}^{h}\right)\right\} .
\end{gathered}
$$

The organization of this paper is as follows. In Section 2, we give some basic definitions and necessary lemmas which will be used in later sections. In Sections 3 and 4, by using a fixed point theorem and constructing suitable Lyapunov functional, we obtain some sufficient conditions ensuring existence, uniqueness, and global exponential stability of almost periodic solution of system (1). Finally, an example is given to illustrate that our results are feasible.

\section{Preliminaries}

Now, let us state the following definitions and lemmas, which will be useful in proving our main result.

Definition 1 (see [14]). $x \in C(\mathbb{R})$ is called almost periodic, if for any $\epsilon>0$ it is possible to find a real number $l=l(\epsilon)>0$ and for any interval with length $l(\epsilon)$ there exists a number $\tau=\tau(\epsilon)$ in this interval such that $|x(t+\tau)-x(t)|<\epsilon, \forall t \in \mathbb{R}$. The collection of those functions is denoted by $A P(\mathbb{R})$.
Definition 2 (see [14]). Let $y \in C\left(\mathbb{R}, \mathbb{R}^{n}\right)$ and let $P(t)$ be a $n \times n$ continuous matrix defined on $\mathbb{R}$. The linear system

$$
\dot{y}(t)=P(t) y(t)
$$

is said to be an exponential dichotomy on $\mathbb{R}$ if there exist constants $k, \lambda>0$, projection $S$, and the fundamental matrix $Y(t)$ satisfying

$$
\begin{gathered}
\left\|Y(t) S Y^{-1}(s)\right\| \leq k e^{-\lambda(t-s)}, \quad \forall t \geq s, \\
\left\|Y(t)(I-S) Y^{-1}(s)\right\| \leq k e^{-\lambda(s-t)}, \quad \forall t \leq s .
\end{gathered}
$$

Lemma 3 (see [14]). If the linear system $\dot{y}(t)=P(t) y(t)$ has an exponential dichotomy, then almost periodic system

$$
\dot{y}(t)=P(t) y(t)+g(t)
$$

has a unique almost periodic solution $y(t)$ which can be expressed as follows:

$$
\begin{aligned}
y(t)= & \int_{-\infty}^{t} Y(t) S Y^{-1}(s) g(s) \mathrm{d} s \\
& -\int_{t}^{\infty} Y(t)(I-S) Y^{-1}(s) g(s) \mathrm{d} s .
\end{aligned}
$$

Lemma 4 (see [14]). Let $a_{i}(t)$ be an almost periodic function and

$$
M\left[a_{i}\right]=\lim _{T \rightarrow \infty} \frac{1}{T} \int_{t}^{t+T} a_{i}(s) \mathrm{d} s>0, \quad \mathrm{i}=1,2, \ldots, \mathrm{n} .
$$

Then the linear system $\dot{y}(t)=-A(t) y(t)$ admits an exponential dichotomy, where $A(t)=\operatorname{diag}\left\{a_{1}(t), a_{2}(t), \ldots, a_{n}(t)\right\}$.

Definition 5. The almost periodic solution $z=\left(x_{1}, \ldots, x_{n}\right)^{T}$ of system (1) with the initial value $z^{*}=\left(x_{1}^{*}, \ldots, x_{n}^{*}\right)^{T}$ is said to be globally exponentially stable, if there exist constants $\omega>0$ and $M \geq 1$, for any solution $\phi=\left(\varphi_{1}, \ldots, \varphi_{n}\right)^{T}$ of system (1) with initial value $\phi^{*}=\left(\varphi_{1}^{*}, \ldots, \varphi_{n}^{*}\right)^{T}$ such that

$$
\sum_{i=1}^{n}\left|x_{i}(t)-\varphi_{i}(t)\right| \leq M\left\|z^{*}-\phi^{*}\right\|_{\infty} e^{-\omega t}, \quad \forall t>0,
$$

where

$$
\begin{aligned}
&\left\|z^{*}-\phi^{*}\right\|_{\infty} \\
& \quad:=\max _{1 \leq i \leq n} \sup _{s \in(-\infty, 0]}\left\{\left|x_{i}^{*}(s)-\varphi_{i}^{*}(s)\right|+\left|\dot{x}_{i}^{*}(s)-\dot{\varphi}_{i}^{*}(s)\right|\right\} .
\end{aligned}
$$

Lemma 6 (see $[15,16])$. Let $(\mathbb{X},\|\cdot\|)$ be a Banach space. Assume that $0 \in \Omega$ is an open bounded subset of $\mathbb{X} ; T: \bar{\Omega} \rightarrow$ $\mathbb{X}$ is completely continuous satisfying

$$
\|T x\| \leq\|x\|, \quad \forall x \in \partial \Omega
$$

then $T$ has a fixed point in $\bar{\Omega}$. 
Let $\mathbb{E}=\left\{x \in A P(\mathbb{R}) \cap C^{1}(\mathbb{R}): \dot{x} \in A P(\mathbb{R})\right\}$ and

$$
\mathbb{X}=\left\{z=\left(x_{1}, \ldots, x_{n}\right)^{T}: x_{i} \in \mathbb{E}, i=1, \ldots, n\right\}
$$

with the norm

$$
\begin{aligned}
\|z\| & =\max \left\{\|z\|_{0},\|\dot{z}\|_{0}\right\} \\
& =\max \left\{\max _{1 \leq i \leq n}\left\{\left|x_{i}\right|_{0}\right\}, \max _{1 \leq i \leq n}\left\{\left|\dot{x}_{i}\right|_{0}\right\}\right\},
\end{aligned}
$$

where $\|z\|_{0}=\max _{1 \leq i \leq n}\left\{\left|x_{i}\right|_{0}\right\},\|\dot{z}\|_{0}=\max _{1 \leq i \leq n}\left\{\left|\dot{x}_{i}\right|_{0}\right\},|f|_{0}=$ $\sup _{s \in \mathbb{R}}|f(s)|, \forall f \in A P(\mathbb{R})$. Then $\mathbb{X}$ is a Banach space with the norm $\|\cdot\|$.

Let $\rho=\max \left\{\rho_{1}, \rho_{2}\right\}$, where

$$
\begin{array}{r}
\rho_{1}=\max _{1 \leq i \leq n}\left\{( a _ { i } ^ { - } ) ^ { - 1 } \left[\sum_{j=1}^{n} b_{i j}^{+} f_{j}(0)+\sum_{j=1}^{n} c_{i j}^{+} g_{j}(0)\right.\right. \\
\left.\left.+\sum_{j=1}^{n} d_{i j}^{+} h_{j}(0)+I_{i}^{+}\right]\right\}, \\
\rho_{2}=\max _{1 \leq i \leq n}\left\{[ 1 + \frac { a _ { i } ^ { u } } { a _ { i } ^ { l } } ] \left[\sum_{j=1}^{n} b_{i j}^{+} f_{j}(0)+\sum_{j=1}^{n} c_{i j}^{+} g_{j}(0)\right.\right. \\
\left.\left.+\sum_{j=1}^{n} d_{i j}^{+} h_{j}(0)+I_{i}^{+}\right]\right\} .
\end{array}
$$

Define an open bounded subset $\Omega$ in $\mathbb{X}$ by

$$
\Omega=\left\{z \in \mathbb{X}:\|z\|<r_{0}=\frac{\rho}{1-\theta}\right\} .
$$

By Lemmas 3 and 4, system (1) has a unique almost periodic solution $z^{\phi}=\left(x_{1}^{\varphi_{1}}, \ldots, x_{n}^{\varphi_{n}}\right)^{T}$ which can be expressed as follows:

$$
x_{i}^{\varphi_{i}}=\int_{-\infty}^{t} e^{-\int_{s}^{t} a_{i}(u) \mathrm{d} u} F_{i}(s, \phi(s)) \mathrm{d} s,
$$

where $\phi=\left(\varphi_{1}, \ldots, \varphi_{n}\right)^{T}$,

$$
\begin{aligned}
F_{i}(t, \phi(t))= & \sum_{j=1}^{n} b_{i j}(t) f_{j}\left(\varphi_{j}(t)\right) \\
& +\sum_{j=1}^{n} c_{i j}(t) g_{j}\left(\int_{0}^{\infty} w_{i j}(s) \varphi_{j}(t-s) \mathrm{d} s\right) \\
& +\sum_{j=1}^{n} d_{i j}(t) h_{j}\left(\int_{0}^{\infty} v_{i j}(s) \dot{\varphi}_{j}(t-s) \mathrm{d} s\right)+I_{i}(t),
\end{aligned}
$$

where $i=1,2, \ldots, n, \forall t \in \mathbb{R}$.

Let the map $T: \mathbb{X} \rightarrow \mathbb{X}$ be defined by

$$
\begin{array}{r}
T(\phi)=\left(\Phi_{1}(\phi), \ldots, \Phi_{n}(\phi)\right)^{T}=\left(x_{1}^{\varphi_{1}}, \ldots, x_{n}^{\varphi_{n}}\right)^{T}, \\
\forall \phi=\left(\varphi_{1}, \ldots, \varphi_{n}\right)^{T} \in \mathbb{X} .
\end{array}
$$

Lemma 7. $T: \bar{\Omega} \rightarrow \mathbb{X}$ is completely continuous.
Proof. First, we show that $T: \bar{\Omega} \rightarrow \mathbb{X}$ is continuous. Assume that $\phi^{1}=\left(\varphi_{1}^{1}, \ldots, \varphi_{n}^{1}\right)^{T}, \phi^{2}=\left(\varphi_{1}^{2}, \ldots, \varphi_{n}^{2}\right)^{T} \in \Omega$; then

$$
\begin{aligned}
& \left|F_{i}\left(t, \phi^{1}\right)-F_{i}\left(t, \phi^{2}\right)\right| \\
& =\mid \sum_{j=1}^{n} b_{i j}(t)\left[f_{j}\left(\varphi_{j}^{1}(t)\right)-f_{j}\left(\varphi_{j}^{2}(t)\right)\right] \\
& \quad+\sum_{j=1}^{n} c_{i j}(t)\left[g_{j}\left(\int_{0}^{\infty} w_{i j}(s) \varphi_{j}^{1}(t-s) \mathrm{d} s\right)\right. \\
& \quad+\sum_{j=1}^{n} d_{i j}(t)\left[h_{j}\left(\int_{0}^{\infty} v_{i j}(s) \dot{\varphi}_{j}^{1}(t-s) \mathrm{d} s\right)\right. \\
& \left.\quad-h_{j}\left(\int_{0}^{\infty} v_{i j}(s) \dot{\varphi}_{j}^{2}(t-s) \mathrm{d} s\right)\right] \mid \\
& \leq \sum_{j=1}^{n}\left(b_{i j}^{+} L_{j}^{f}+c_{i j}^{+} L_{j}^{g}\right)\left|\varphi_{j}^{1}-\varphi_{j}^{2}\right|_{0} \\
& \quad+\sum_{j=1}^{n} d_{i j}^{+} L_{j}^{h}\left|\dot{\varphi}_{j}^{1}-\dot{\varphi}_{j}^{2}\right|_{0} \\
& \leq \max \left\{\sum_{j=1}^{n} b_{i j}^{+} L_{j}^{f}+c_{i j}^{+} L_{j}^{g}, \sum_{j=1}^{n} d_{i j}^{+} L_{j}^{h}\right\}\left\|\phi^{1}-\phi^{2}\right\|,
\end{aligned}
$$

which implies that

$$
\begin{aligned}
& \left|\Phi_{i}\left(\phi^{1}\right)-\Phi_{i}\left(\phi^{2}\right)\right| \\
& \quad \leq \int_{-\infty}^{t} e^{-\int_{s}^{t} a_{i}(u) \mathrm{d} u}\left|F_{i}\left(s, \phi^{1}(s)\right)-F_{i}\left(s, \phi^{2}(s)\right)\right| \mathrm{d} s \\
& \quad \leq\left(a_{i}^{-}\right)^{-1} \max \left\{\sum_{j=1}^{n} b_{i j}^{+} L_{j}^{f}+c_{i j}^{+} L_{j}^{g}, \sum_{j=1}^{n} d_{i j}^{+} L_{j}^{h}\right\}\left\|\phi^{1}-\phi^{2}\right\| \\
& \quad \leq \sum_{i=1}^{n}\left(a_{i}^{-}\right)^{-1} \max \left\{\sum_{j=1}^{n} b_{i j}^{+} L_{j}^{f}+c_{i j}^{+} L_{j}^{g}, \sum_{j=1}^{n} d_{i j}^{+} L_{j}^{h}\right\}\left\|\phi^{1}-\phi^{2}\right\| \\
& \quad:=l_{1}\left\|\phi^{1}-\phi^{2}\right\|,
\end{aligned}
$$

where $l_{1}=\sum_{i=1}^{n}\left(a_{i}^{-}\right)^{-1} \max \left\{\sum_{j=1}^{n} b_{i j}^{+} L_{j}^{f}+c_{i j}^{+} L_{j}^{g}, \sum_{j=1}^{n} d_{i j}^{+} L_{j}^{h}\right\}, i=$ $1, \ldots, n$. Since

$$
\begin{aligned}
& \left|\dot{\Phi}_{i}\left(\phi^{1}\right)-\dot{\Phi}_{i}\left(\phi^{2}\right)\right| \\
& =\mid\left[-a_{i}(t) \Phi_{i}\left(\phi^{1}\right)+F_{i}\left(t, \phi^{1}\right)\right] \\
& \quad-\left[a_{i}(t) \Phi_{i}\left(\phi^{2}\right)+F_{i}\left(t, \phi^{2}\right)\right] \mid
\end{aligned}
$$




$$
\begin{aligned}
& \leq a_{i}^{+}\left|\Phi_{i}\left(\phi^{1}\right)-\Phi_{i}\left(\phi^{2}\right)\right|+\left|F_{i}\left(t, \phi^{1}\right)-F_{i}\left(t, \phi^{2}\right)\right| \\
& \leq\left(1+\frac{a_{i}^{+}}{a_{i}^{-}}\right) \max \left\{\sum_{j=1}^{n} b_{i j}^{+} L_{j}^{f}+c_{i j}^{+} L_{j}^{g}, \sum_{j=1}^{n} d_{i j}^{+} L_{j}^{h}\right\}\left\|\phi^{1}-\phi^{2}\right\| \\
& :=l_{2}\left\|\phi^{1}-\phi^{2}\right\|,
\end{aligned}
$$

where $l_{2}=\left(1+\left(a_{i}^{+} / a_{i}^{-}\right)\right) \max \left\{\sum_{j=1}^{n} b_{i j}^{+} L_{j}^{f}+c_{i j}^{+} L_{j}^{g}, \sum_{j=1}^{n} d_{i j}^{+} L_{j}^{h}\right\}$, $i=1, \ldots, n$, so

$$
\left\|T\left(\phi^{1}\right)-T\left(\phi^{2}\right)\right\| \leq \sum_{i=1}^{2} l_{i}\left\|\phi^{1}-\phi^{2}\right\| .
$$

For arbitrary $\epsilon>0$, taking $\delta=\left(\epsilon / \sum_{i=1}^{2} l_{i}\right)$, when $\left\|\phi^{1}-\phi^{2}\right\|<\delta$, one has

$$
\left\|T\left(\phi^{1}\right)-T\left(\phi^{2}\right)\right\|<\epsilon
$$

which implies that $T: \bar{\Omega} \rightarrow \mathbb{X}$ is continuous.

Next, we show that $T$ maps bounded set onto itself. Assume $c$ is a positive constant and $\phi \in \bar{\Omega}_{c}=\{z \in \Omega$ : $\|z\| \leq c\}$. By the almost periodicity of system (1), there exists a constant $C>0$ such that

$$
\max _{1 \leq i \leq n, 1 \leq j \leq m} \max _{s \in \mathbb{R}, \phi \in \bar{\Omega}_{c}}\left\{F_{i}(s, 0), F_{i}(s, \phi)\right\}<C .
$$

From the above analysis, we get that

$$
\begin{aligned}
\left|\Phi_{i}(\phi)\right|= & \int_{-\infty}^{t} e^{-\int_{s}^{t} a_{i}(u) \mathrm{d} u}\left|F_{i}(s, \phi(s))-F_{i}(s, 0)\right| \mathrm{d} s \\
& +\int_{-\infty}^{t} e^{-\int_{s}^{t} a_{i}(u) \mathrm{d} u} F_{i}(s, 0) \mathrm{d} s \\
\leq & l_{1}\|\phi\|+\frac{C}{a_{i}^{-}}, \quad i=1, \ldots, n, \\
\left|\dot{\Phi}_{i}(\phi)\right|= & \left|-a_{i}(t) \Phi_{i}(\phi(t))+F_{i}(t, \phi)\right| \\
\leq & a_{i}^{+}\left[l_{1}\|\phi\|+\frac{C}{a_{i}^{-}}\right]+C, \quad i=1, \ldots, n,
\end{aligned}
$$

which imply that $T \bar{\Omega}_{c}$ is uniformly bounded. In addition, notice that

$$
\begin{aligned}
& \left|\Phi_{i}\left(\phi\left(t_{1}\right)\right)-\Phi_{i}\left(\phi\left(t_{2}\right)\right)\right| \\
& \quad=\left|\int_{t_{2}}^{t_{1}} e^{-\int_{s}^{t} a_{i}(u) \mathrm{d} u} F_{i}(s, \phi(s)) \mathrm{d} s\right| \\
& \quad \leq C\left|t_{1}-t_{2}\right|, \quad i=1, \ldots, n .
\end{aligned}
$$

Using Arzela-Ascoli theorem, we obtain that $T \bar{\Omega}_{c}$ is relatively compact. Hence, $T$ is completely continuous. The proof of this lemma is complete.

\section{Existence of Almost Periodic Solution}

In this section, we study the existence of almost periodic solutions of system (1).

Theorem 8. Assume that $\left(H_{1}\right)-\left(H_{3}\right)$ hold; then system (1) admits at least one almost periodic solution.

Proof. Consider the following nonlinear operator:

$$
T(\phi)=\left(\Phi_{1}(\phi), \ldots, \Phi_{n}(\phi)\right)^{T}, \quad \forall \phi=\left(\varphi_{1}, \ldots, \varphi_{n}\right)^{T} \in \bar{\Omega} .
$$

For $\forall \phi=\left(\varphi_{1}, \ldots, \varphi_{n}, \psi_{1}, \ldots, \psi_{m}\right)^{T} \in \partial \Omega$ we have $\|\phi\|=r_{0}$. From Lemma 7, $T: \bar{\Omega} \rightarrow \mathbb{X}$ is completely continuous. Similar to the arguments as that in Lemma 7, it follows from the definition of $F_{i}$ and $G_{j}$ that

$$
\begin{aligned}
\left|F_{i}\right|_{0}= & \sup _{s \in \mathbb{R}}\left|F_{i}(s, \phi)\right| \\
\leq & \sup _{s \in \mathbb{R}}\left|F_{i}(s, \phi)-F_{i}(s, 0)\right|+\sup _{s \in \mathbb{R}}\left|F_{i}(s, 0)\right| \\
\leq & \sum_{j=1}^{n}\left(b_{i j}^{+} L_{j}^{f}+c_{i j}^{+} L_{j}^{g}\right)\left|\varphi_{j}\right|_{0}+\sum_{j=1}^{n} d_{i j}^{+} L_{j}^{h}\left|\dot{\varphi}_{j}\right|_{0} \\
& +\sum_{j=1}^{n} b_{i j}^{+} f_{j}(0)+\sum_{j=1}^{n} c_{i j}^{+} g_{j}(0)+\sum_{j=1}^{n} d_{i j}^{+} h_{j}(0)+I_{i}^{+},
\end{aligned}
$$

which yields that

$$
\begin{aligned}
\left|\Phi_{i}(\phi)\right|_{0}= & \sup _{t \in \mathbb{R}}\left|\Phi_{i}(\phi(t))\right| \\
\leq & \sup _{t \in \mathbb{R}}\left|\int_{-\infty}^{t} e^{-\int_{s}^{t} a_{i}(u) \mathrm{d} u} F_{i}(s, \phi(s)) \mathrm{d} s\right| \\
\leq & \left(a_{i}^{-}\right)^{-1} \sum_{j=1}^{n}\left(b_{i j}^{+} L_{j}^{f}+c_{i j}^{+} L_{j}^{g}+d_{i j}^{+} L_{j}^{h}\right)\|\phi\| \\
& +\left(a_{i}^{-}\right)^{-1}\left[\sum_{j=1}^{n} b_{i j}^{+} f_{j}(0)+\sum_{j=1}^{n} c_{i j}^{+} g_{j}(0)\right. \\
\left|\dot{\Phi}_{i}(\phi)\right|_{0}= & \sup _{t \in \mathbb{R}}\left|\dot{\Phi}_{i}(\phi(t))\right| \\
= & \left.\sup _{t \in \mathbb{R}} \mid-a_{i}(t) \Phi_{j=1}^{n} d_{i j}^{+} h_{j}(0)+I_{i}^{+}\right] \\
\leq & {\left[1+\frac{a_{i}^{u}}{a_{i}^{l}}\right] \sum_{j=1}^{n}\left(b_{i j}^{+} L_{j}^{f}+c_{i j}^{+} L_{j}^{g}+d_{i j}^{+} L_{j}^{h}\right)\|\phi\| } \\
& +\left[1+\frac{a_{i}^{u}}{a_{i}^{l}}\right]\left[\sum_{j=1}^{n} b_{i j}^{+} f_{j}(0)+\sum_{j=1}^{n} c_{i j}^{+} g_{j}(0)\right. \\
& \left.+\sum_{j=1}^{n} d_{i j}^{+} h_{j}(0)+I_{i}^{+}\right] \\
& \\
& \\
& \\
&
\end{aligned}
$$


where $i=1,2, \ldots, n$. Hence,

$$
\begin{aligned}
&\|T(\phi)\|_{0}= \max _{1 \leq i \leq n}\left\{\left|\Phi_{i}(\phi)\right|_{0}\right\} \\
& \leq \max _{1 \leq i \leq n}\left\{\left(a_{i}^{-}\right)^{-1} \sum_{j=1}^{n}\left(b_{i j}^{+} L_{j}^{f}+c_{i j}^{+} L_{j}^{g}+d_{i j}^{+} L_{j}^{h}\right)\right\}\|\phi\| \\
&+\max _{1 \leq i \leq n}\left\{( a _ { i } ^ { - } ) ^ { - 1 } \left[\sum_{j=1}^{n} b_{i j}^{+} f_{j}(0)+\sum_{j=1}^{n} c_{i j}^{+} g_{j}(0)\right.\right. \\
&\left.\left.+\sum_{j=1}^{n} d_{i j}^{+} h_{j}(0)+I_{i}^{+}\right]\right\} \\
&= \theta_{1} r_{0}+\rho_{1}, \\
&\|\dot{T}(\phi)\|_{0}= \max _{1 \leq i \leq n}\left\{\left|\dot{\Phi}_{i}(\phi)\right|_{0}\right\} \\
& \leq \max _{1 \leq i \leq n}\left\{\left[1+\frac{a_{i}^{u}}{a_{i}^{l}}\right] \sum_{j=1}^{n}\left(b_{i j}^{+} L_{j}^{f}+c_{i j}^{+} L_{j}^{g}+d_{i j}^{+} L_{j}^{h}\right)\right\}\|\phi\| \\
&+\max _{1 \leq i \leq n}\left\{[ 1 + \frac { a _ { i } ^ { u } } { a _ { i } ^ { l } } ] \left[\sum_{j=1}^{n} b_{i j}^{+} f_{j}(0)+\sum_{j=1}^{n} c_{i j}^{+} g_{j}(0)\right.\right. \\
&= \theta_{2} r_{0}+\rho_{2} . \\
&\left.\left.+\sum_{j=1}^{n} d_{i j}^{+} h_{j}(0)+I_{i}^{+}\right]\right\}
\end{aligned}
$$

So

$$
\begin{aligned}
\|T(\phi)\| & =\max \left\{\|T(\phi)\|_{0},\|\dot{T}(\phi)\|_{0}\right\} \\
& \leq \max \left\{\theta_{1}, \theta_{2}\right\}\|\phi\|+\max \left\{\rho_{1}, \rho_{2}\right\} \\
& =\theta r_{0}+\rho \\
& \leq \frac{\rho}{1-\theta} \\
& =r_{0} \\
& =\|\phi\| .
\end{aligned}
$$

By Lemma 6, there exists at least a fixed point $\phi_{0} \in \bar{\Omega}$ satisfying $T\left(\phi_{0}\right)=\phi_{0}$, which implies system (1) has at least one almost periodic solution. This completes the proof.

\section{Globally Exponential Stability of Almost Periodic Solution}

Theorem 9. Assume that $\left(H_{1}\right)-\left(H_{3}\right)$ hold; suppose further the following.
$\left(H_{4}\right)$ There exists a positive constant $\lambda<1$ such that

$$
\begin{aligned}
& \sup _{s \in \mathbb{R}}\left\{\sum _ { j = 1 } ^ { n } \left[\left|b_{j i}(s)\right| L_{i}^{f}+(1+\lambda) L_{i}^{g}\right.\right. \\
& \left.\left.\quad \times \int_{0}^{\infty} w_{i}(\mu)\left|c_{j i}(s+\mu)\right| d \mu\right]-(1-\lambda) a_{i}(s)\right\} \\
& <0, \\
& \sup _{s \in \mathbb{R}}\left\{[1+\lambda] \sum_{j=1}^{n}\left|d_{j i}(s)\right| L_{i}^{h}-\lambda\right\}<0, \quad i=1,2, \ldots, n .
\end{aligned}
$$

Then system (1) has a unique almost periodic solution, which is globally exponentially stable.

Proof. It follows from Theorem 8 that system (1) has at least one almost periodic solution $\phi=\left(\varphi_{1}, \ldots, \varphi_{n}\right)^{T}$ with initial value $\phi^{*}=\left(\varphi_{1}^{*}, \ldots, \varphi_{n}^{*}\right)^{T}$. We next show that the almost periodic solution $\phi$ is globally exponentially stable.

Make a transformation for system (1): $x_{i}=u_{i}-\varphi_{i}, x_{i}^{*}=$ $u_{i}^{*}-\varphi_{i}^{*}, i=1,2, \ldots, n$, where $z=\left(u_{1}, \ldots, u_{n}\right)^{T}$ is arbitrary solution of system (1) with initial value $z^{*}=\left(u_{1}^{*}, \ldots, u_{n}^{*}\right)^{T}$.

By (2) and $\left(H_{4}\right)$, there exist small enough positive constants $\omega \leq \delta$ and $\epsilon$ such that

$$
\begin{gathered}
\int_{0}^{\infty} v_{i}(\nu) e^{\omega \nu} \mathrm{d} \nu \leq \int_{0}^{\infty} v_{i}(\nu)(1+\epsilon) \mathrm{d} v=(1+\epsilon), \\
\int_{0}^{\infty} w_{j}(\mu)\left|c_{i j}(s+\mu)\right| e^{\omega \mu} \mathrm{d} \mu \\
\leq(1+\epsilon) \int_{0}^{\infty} w_{j}(\mu)\left|c_{i j}(s+\mu)\right| \mathrm{d} \mu \\
\omega+\sum_{j=1}^{n}\left(\left|b_{j i}(s)\right| L_{i}^{f}+[1+\lambda(1+\epsilon)](1+\epsilon) L_{i}^{g}\right. \\
\left.\times \int_{0}^{\infty} w_{i}(\mu)\left|c_{j i}(s+\mu)\right| \mathrm{d} \mu\right) \\
\leq[1-\lambda(1+\epsilon)] a_{i}(s), \\
{[1+\lambda(1+\epsilon)] \sum_{j=1}^{n}\left|d_{j i}(s)\right| L_{i}^{h}-\lambda \leq 0}
\end{gathered}
$$

where $i, j=1,2, \ldots, n, s \in \mathbb{R}$.

Define

$$
V_{1}(t)=\sum_{i=1}^{n} e^{\omega t}\left|x_{i}(t)\right|
$$


In view of system (1), we have

$$
\begin{aligned}
& D^{+} V_{1}(t) \\
& \leq \omega \sum_{i=1}^{n} e^{\omega t}\left|x_{i}(t)\right| \\
& +\sum_{i=1}^{n} e^{\omega t}\left[\begin{array}{l}
-a_{i}(t)\left|x_{i}(t)\right|+\sum_{j=1}^{n}\left|b_{i j}(t)\right| L_{j}^{f}\left|x_{j}(t)\right| \\
+\sum_{j=1}^{n}\left|c_{i j}(t)\right| L_{j}^{g} \int_{0}^{\infty} w_{j}(\mu)\left|x_{j}(t-\mu)\right| \mathrm{d} \mu \\
\left.+\sum_{j=1}^{n}\left|d_{i j}(t)\right| L_{j}^{h} \int_{0}^{\infty} v_{j}(\nu)\left|\dot{x}_{j}(t-\nu)\right| \mathrm{d} \nu\right] .
\end{array}\right.
\end{aligned}
$$

Let

$$
\begin{aligned}
V_{2}(t)= & \lambda \sum_{j=1}^{n} \int_{0}^{\infty} v_{j}(\nu) \int_{t-\nu}^{t} e^{\omega(r+v)}\left|\dot{x}_{j}(r)\right| \mathrm{d} r \mathrm{~d} \nu \\
V_{3}(t)= & \sum_{i=1}^{n} \sum_{j=1}^{n}[1+\lambda(1+\epsilon)] L_{j}^{g} \\
& \times \int_{0}^{\infty} w_{j}(\mu) \int_{t-\mu}^{t}\left|c_{i j}(r+\mu)\right| e^{\omega(r+\mu)}\left|x_{j}(r)\right| \mathrm{d} r \mathrm{~d} \mu .
\end{aligned}
$$

So

$$
\begin{aligned}
& D^{+} V_{2}(t) \\
& \leq \lambda \sum_{j=1}^{n} e^{\omega t} \int_{0}^{\infty} v_{j}(\nu) e^{\omega \nu} \mathrm{d} \nu\left|\dot{x}_{j}(t)\right| \\
& -\lambda \sum_{j=1}^{n} e^{\omega t} \int_{0}^{\infty} v_{j}(\nu)\left|\dot{x}_{j}(t-v)\right| \mathrm{d} v \\
& \leq \lambda \sum_{i=1}^{n} e^{\omega t}(1+\epsilon)\left[\begin{array}{c}
a_{i}(t)\left|x_{i}(t)\right| \\
+\sum_{j=1}^{n}\left|b_{i j}(t)\right| L_{j}^{f}\left|x_{j}(t)\right| \\
+\sum_{j=1}^{n}\left|c_{i j}(t)\right| L_{j}^{g} \\
\left.\times \int_{0}^{\infty} w_{j}(\mu)\left|x_{j}(t-\mu)\right| \mathrm{d} \mu\right]
\end{array}\right.
\end{aligned}
$$

$$
\begin{aligned}
& -\lambda \sum_{j=1}^{n} e^{\omega t}\left[1-\sum_{i=1}^{n}(1+\epsilon)\left|d_{i j}(t)\right| L_{j}^{h}\right] \\
& \times \int_{0}^{\infty} v_{j}(v)\left|\dot{x}_{j}(t-v)\right| \mathrm{d} \nu
\end{aligned}
$$$$
D^{+} V_{3}(t) \leq \sum_{i=1}^{n} \sum_{j=1}^{n}[1+\lambda(1+\epsilon)] e^{\omega t} L_{j}^{g}
$$$$
\times \int_{0}^{\infty} w_{j}(\mu)\left|c_{i j}(t+\mu)\right| e^{\omega \mu} \mathrm{d} \mu\left|x_{j}(t)\right|
$$$$
-\sum_{i=1}^{n} \sum_{j=1}^{n}[1+\lambda(1+\epsilon)] e^{\omega t}\left|c_{i j}(t)\right| L_{j}^{g}
$$$$
\times \int_{0}^{\infty} w_{j}(\mu)\left|x_{j}(t-\mu)\right| \mathrm{d} \mu
$$$$
\leq \sum_{i=1}^{n} \sum_{j=1}^{n}[1+\lambda(1+\epsilon)](1+\epsilon) e^{\omega t} L_{j}^{g}
$$$$
\times \int_{0}^{\infty} w_{j}(\mu)\left|c_{i j}(t+\mu)\right| \mathrm{d} \mu\left|x_{j}(t)\right|
$$$$
-\sum_{i=1}^{n} \sum_{j=1}^{n}[1+\lambda(1+\epsilon)] e^{\omega t}\left|c_{i j}(t)\right| L_{j}^{g}
$$$$
\times \int_{0}^{\infty} w_{j}(\mu)\left|x_{j}(t-\mu)\right| \mathrm{d} \mu .
$$

$$
\text { Let } V(t)=\sum_{q=1}^{3} V_{q} \text {. From (38)-(41), it follows that }
$$

$$
\begin{aligned}
D^{+} V(t) \leq e^{\omega t} \sum_{i=1}^{n}\{\omega & -[1-\lambda(1+\epsilon)] a_{i}(t) \\
& +\sum_{j=1}^{n}\left|b_{j i}(t)\right| L_{i}^{f} \\
& +\sum_{j=1}^{n}[1+\lambda(1+\epsilon)](1+\epsilon) L_{i}^{g} \\
& \left.\times \int_{0}^{\infty} w_{i}(\mu)\left|c_{j i}(t+\mu)\right| \mathrm{d} \mu\right\}\left|x_{i}(t)\right| \\
+ & e^{\omega t} \sum_{j=1}^{n}\left\{[1+\lambda(1+\epsilon)] \sum_{i=1}^{n}\left|d_{i j}(t)\right| L_{j}^{h}-\lambda\right\} \\
& \times \int_{0}^{\infty} v_{j}(\nu)\left|\dot{x}_{j}(t-v)\right| \mathrm{d} \nu \leq 0,
\end{aligned}
$$

which implies that $V(t) \leq V(0), \forall t>0$. Obviously,

$$
\sum_{i=1}^{n} e^{\omega t}\left|x_{i}(t)\right| \leq V(t) .
$$


On the other hand, we have

$$
\begin{aligned}
V(0)= & \sum_{i=1}^{n}\left|x_{i}(0)\right| \\
& +\lambda \sum_{j=1}^{n} \int_{0}^{\infty} v_{j}(\nu) \int_{-\nu}^{0} e^{\omega(r+\nu)}\left|\dot{x}_{j}(r)\right| \mathrm{d} r \mathrm{~d} \nu \\
& +\sum_{i=1}^{n} \sum_{j=1}^{n}[1+\lambda(1+\epsilon)] L_{j}^{g} \\
& \times \int_{0}^{\infty} w_{j}(\mu) \int_{-\mu}^{0}\left|c_{i j}(r+\mu)\right| e^{\omega(r+\mu)}\left|x_{j}(r)\right| \mathrm{d} r \mathrm{~d} \mu \\
\leq & \left\{n+\frac{2 n}{\omega}+\sum_{j=1}^{m} \sum_{i=1}^{n} \frac{6 c_{i j}^{+} L_{j}^{g}}{\omega}\right\}\left\|z^{*}-\phi^{*}\right\|,
\end{aligned}
$$

which implies from (43) that

$$
\sum_{i=1}^{n}\left|x_{i}(t)\right| \leq M\left\|z^{*}-\phi^{*}\right\| e^{-\omega t}, \quad \forall t>0,
$$

where

$$
M:=n+\frac{2 n}{\omega}+\sum_{j=1}^{m} \sum_{i=1}^{n} \frac{6 c_{i j}^{+} L_{j}^{g}}{\omega} .
$$

Thus, the almost periodic solution of system (1) is globally exponentially stable. The globally exponential stability implies that the almost periodic solution is unique. This completes the proof.

\section{An Example}

Example 1. Consider the following neutral-type cellular neural networks with distributed delays:

$$
\begin{aligned}
\dot{x}_{i}(t)= & -x_{i}(t)+\sin (\sqrt{2} t) \\
& \times \sum_{j=1}^{2} g_{j}\left(\int_{0}^{\infty} e^{-s} x_{j}(t-s) \mathrm{d} s\right) \\
& +0.1\left[2+\cos ^{2}(\sqrt{3} t)\right] \sum_{j=1}^{2} h_{j}\left(\int_{0}^{\infty} e^{-s} \dot{x}_{j}(t-s) \mathrm{d} s\right) \\
& +\sin (\sqrt{2} t), \quad i=1,2,
\end{aligned}
$$

where $g_{1}(s)=g_{2}(s)=0.1 s, h_{1}(s)=0.1 \sin (s)$, and $h_{2}(s)=$ $0.1 \cos (s), \forall s \in \mathbb{R}$. Then system (47) has a unique almost periodic solution, which is globally exponentially stable.

Proof. Corresponding to system (1), $a_{i}^{-}=1, L_{j}^{g}=L_{j}^{h}=$ $0.1, c_{i j}^{+}=1, d_{i j}^{+}=0.3, w_{j}(s)=v_{j}(s)=e^{-s}, i, j=1,2$. Taking $\lambda=0.5$, it is easy to verify that $\left(H_{1}\right)-\left(H_{4}\right)$ hold and the results follow from Theorems 8 and 9 (see Figures 1, 2, and 3). This completes the proof.

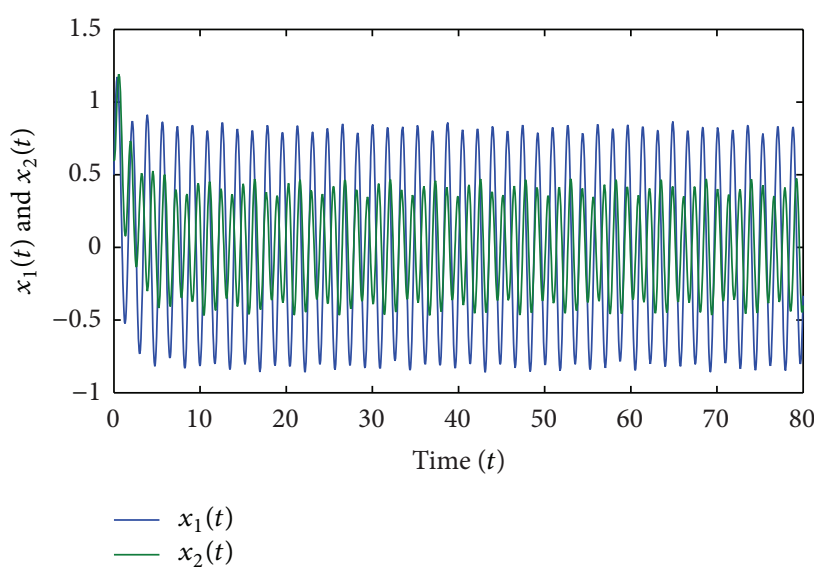

FIgURE 1: Almost periodicity of state variables $x_{1}(t)$ and $x_{2}(t)$.

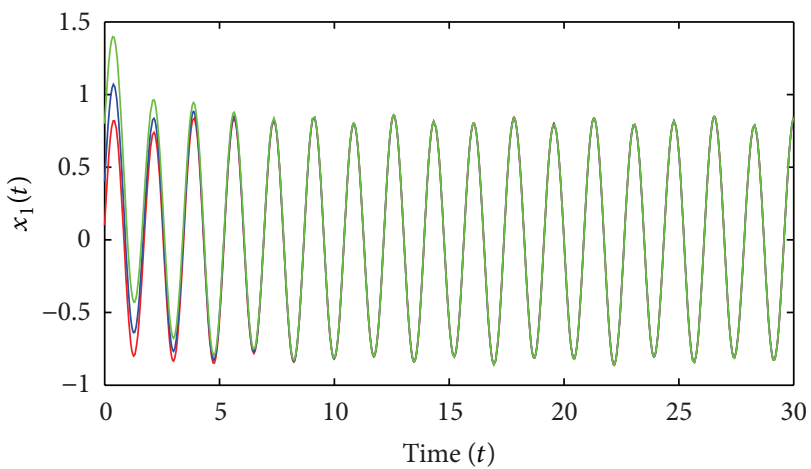

Figure 2: Global exponential stability of state variable $x_{1}(t)$.

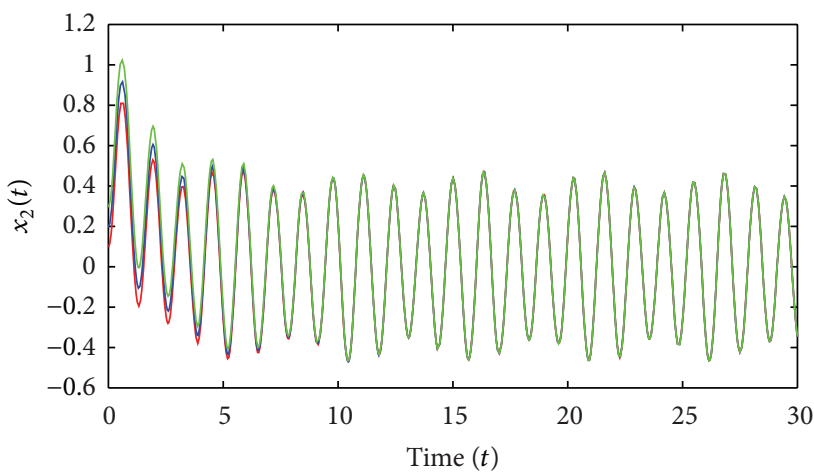

FIgURE 3: Global exponential stability of state variable $x_{2}(t)$.

Remark 10. One can observe that all the results in [9-12] and the references therein cannot be applicable to system (1) to obtain the existence and exponential stability of the almost periodic solutions. This implies that the results of this paper are essentially new.

\section{Conclusion}

This paper is concerned with the neutral cellular neural networks with distributed delays. By using fixed point theory 
and constructing suitable Lyapunov functional, some new sufficient conditions are obtained for the existence and global exponential stability of almost periodic solution of the system. Conditions $\left(H_{3}\right)$ and $\left(H_{4}\right)$ in Theorems 8 and 9 indicate that the neutral terms and the distributed delays are harm for the existence and global exponential stability of almost periodic solution of the neutral-type system. The method used in this paper provides a possible method to study the existence and global exponential stability of almost periodic solution of other neutral neural networks.

\section{Conflict of Interests}

The author declares that there is no conflict of interests regarding the publication of this paper.

\section{Acknowledgment}

The author thanks the referee for his/her careful reading of the original manuscript and valuable comments and suggestions that greatly improved the presentation of this work.

\section{References}

[1] Y. Yang and J. Cao, "Stability and periodicity in delayed cellular neural networks with impulsive effects," Nonlinear Analysis: Real World Applications, vol. 8, no. 1, pp. 362-374, 2007.

[2] Z. Gui and W. Ge, "Periodic solutions of nonautonomous cellular neural networks with impulses," Chaos, Solitons \& Fractals, vol. 32, no. 5, pp. 1760-1771, 2007.

[3] Z. J. Gui and W. G. Ge, "Existence and uniqueness of periodic solutions of nonautonomous cellular neural networks with impulses," Physics Letters A, vol. 354, pp. 84-94, 2006.

[4] J. Zhang and Z. Gui, "Periodic solutions of nonautonomous cellular neural networks with impulses and delays," Nonlinear Analysis: Real World Applications, vol. 10, no. 3, pp. 1891-1903, 2009.

[5] Z. Huang, S. Mohamad, and C. Feng, "New results on exponential attractivity of multiple almost periodic solutions of cellular neural networks with time-varying delays," Mathematical and Computer Modelling, vol. 52, no. 9-10, pp. 1521-1531, 2010.

[6] Y. Li, L. Zhu, and P. Liu, "Existence and stability of periodic solutions of delayed cellular neural networks," Nonlinear Analysis: Real World Applications, vol. 7, no. 2, pp. 225-234, 2006.

[7] Y. Li and Z. Xing, "Existence and global exponential stability of periodic solution of CNNs with impulses," Chaos, Solitons \& Fractals, vol. 33, no. 5, pp. 1686-1693, 2007.

[8] Y. Li, L. Zhao, and X. Chen, "Existence of periodic solutions for neutral type cellular neural networks with delays," Applied Mathematical Modelling, vol. 36, no. 3, pp. 1173-1183, 2012.

[9] A. Chen and J. Cao, "Existence and attractivity of almost periodic solutions for cellular neural networks with distributed delays and variable coefficients," Applied Mathematics and Computation, vol. 134, no. 1, pp. 125-140, 2003.

[10] H. Zhao, "Existence and global attractivity of almost periodic solution for cellular neural network with distributed delays," Applied Mathematics and Computation, vol. 154, no. 3, pp. 683695, 2004.
[11] B. Liu and L. Huang, "Existence and exponential stability of almost periodic solutions for cellular neural networks with mixed delays," Chaos, Solitons and Fractals, vol. 32, no. 1, pp. 95-103, 2007.

[12] W. Zhao and H. Zhang, "New results of almost periodic solutions for cellular neural networks with mixed delays," Chaos, Solitons and Fractals, vol. 40, no. 2, pp. 831-838, 2009.

[13] H. He and X. Liao, "Stability analysis of neutral neural networks with time delay," in Advances in Neural Networks-ISNN 2006, vol. 3971 of Lecture Notes in Computer Science, pp. 147-152, 2006.

[14] A. M. Fink, Almost Periodic Differential Equations, Springer, Berlin, Germany, 1974.

[15] M. Altman, "A fixed point theorem in Hilbert space," Bulletin of the Polish Academy of Sciences, vol. 5, pp. 19-22, 1957.

[16] D. J. Guo, Nonlinear Functional Analysis, Shandong Science and Technology Press, Shandong, China, 2003 (Chinese). 


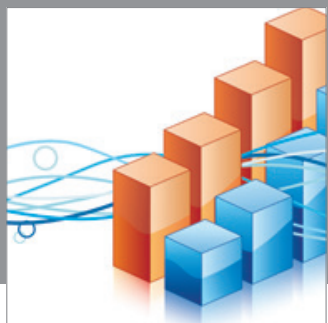

Advances in

Operations Research

mansans

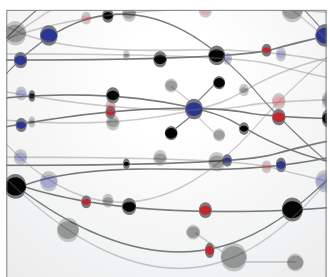

The Scientific World Journal
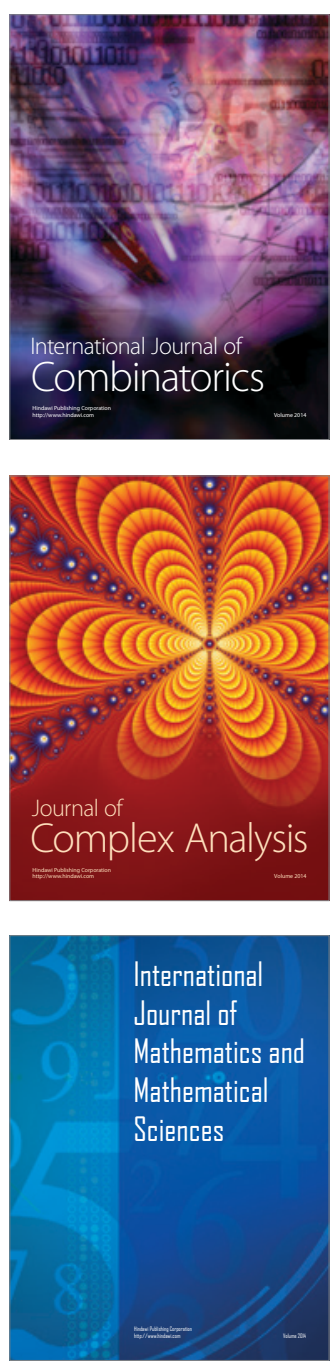
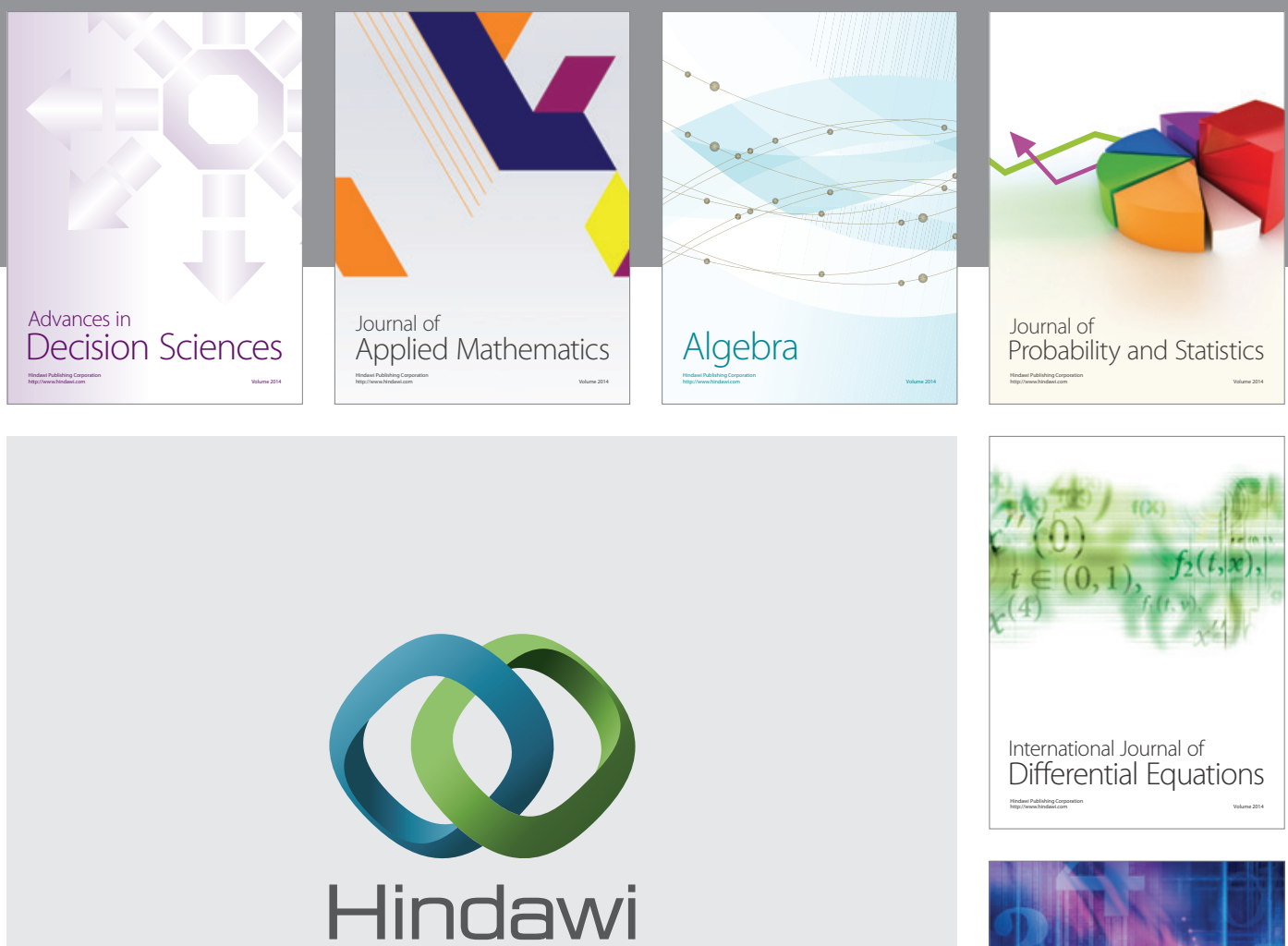

Submit your manuscripts at http://www.hindawi.com
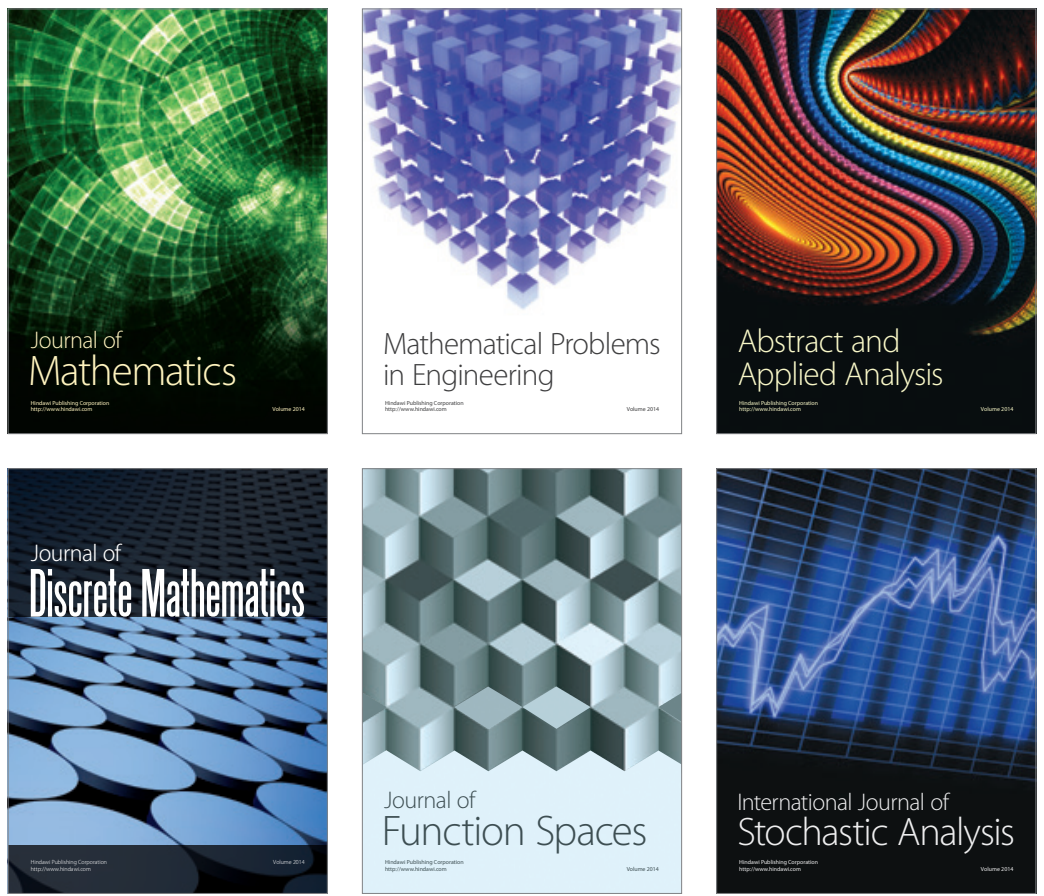

Journal of

Function Spaces

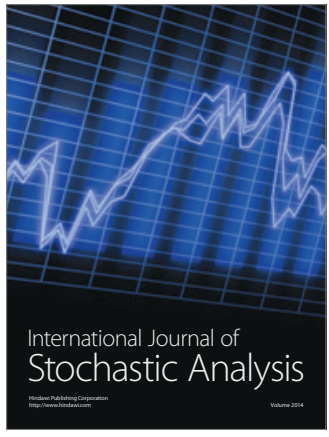

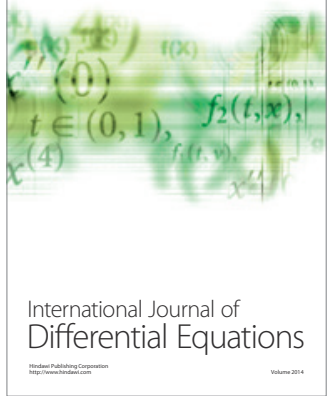
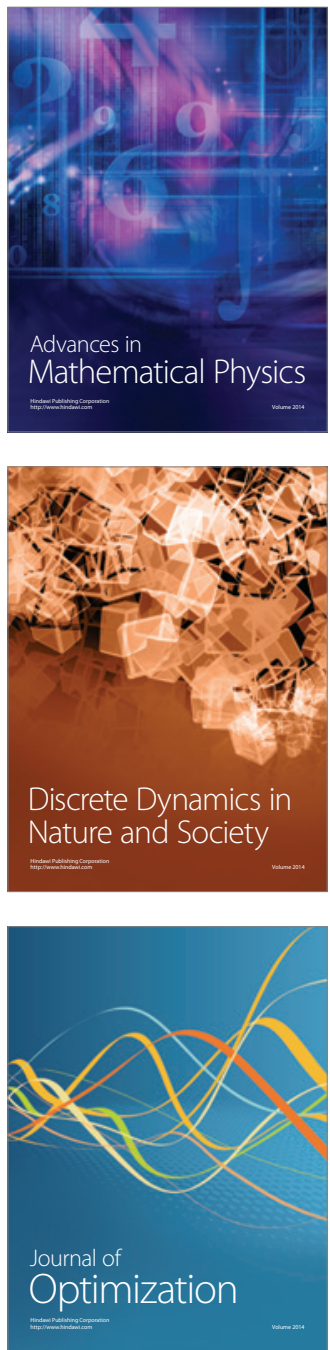